\title{
Infestation and Identification of Ixodid Tick in Cattle: The Case of Arbegona District, Southern Ethiopia
}

\author{
Jelalu Kemal, ${ }^{1}$ Nateneal Tamerat, ${ }^{1}$ and Temesgen Tuluka ${ }^{2}$ \\ ${ }^{1}$ Haramaya University, College of Veterinary Medicine, P.O. Box 138, Dire Dawa, Ethiopia \\ ${ }^{2}$ Sidama Zone Agricultural and Rural Development Office, Southern Ethiopia, Ethiopia \\ Correspondence should be addressed to Jelalu Kemal; jelaluk@gmail.com
}

Received 8 August 2016; Revised 27 October 2016; Accepted 15 November 2016

Academic Editor: Antonio Ortega-Pacheco

Copyright (c) 2016 Jelalu Kemal et al. This is an open access article distributed under the Creative Commons Attribution License, which permits unrestricted use, distribution, and reproduction in any medium, provided the original work is properly cited.

\begin{abstract}
The study was conducted from October 2014 to June 2015 to estimate tick prevalence and identify major tick genera infesting cattle and the associated risk factors in Arbegona district, southern Ethiopia. A total of 2024 adult ticks were collected from main body parts of animals and eight species of ticks which belong to three genera were identified. Questionnaire survey was employed concerning the general case on the tick infestation problems on the cattle. From 384 cattle examined, 291 (75.7\%) were found to be infested with one or more types of tick species. The relative prevalence of each genera was Amblyomma (34.9\%), Rhipicephalus (Boophilus) (26.6\%), Hyalomma (19.2\%), and Rhipicephalus (19\%). The prevalence of tick infestation in good (65.5\%), medium (74\%), and poor body condition animal $(100 \%)$ was found to be statistically significant $(p<0.05)$. There was also significantly $(p<0.05)$ higher prevalence in old $(98.4 \%)$ than adult $(78.8 \%)$ and young $(59.8 \%)$ age groups of animals. In the survey, $87.5 \%$ of respondents believe that there was tick infestation problem in their locality. This study showed there was high burden and prevalence of ticks that still play major roles in reducing productivity and cause health problems of cattle in the area which call for urgent attention.
\end{abstract}

\section{Introduction}

Ethiopia has Africa's largest livestock record with an estimated total cattle population of 57.83 million [1]. Currently, livestock has been contributing to the livelihoods of estimated $80 \%$ of the rural human population of the country [2]. The current utilization of hides and skins is estimated to be $48 \%$ for cattle which accounts for $12-16 \%$ of the total value of exports in the country [3]. However, the contribution from this huge livestock resource to the national income of the country is disproportionately small due to several factors. Ticks are a global problem and considered as a major obstacle in the health and livestock productivity that cause considerable economic losses [2, 4]. A conservative estimate of USD 45,269.35 (1 million ETB) loss annually was made through rejection and downgrading of hides and skins in Ethiopia $[5,6]$.

According to Walker et al. [7] ticks in Africa with veterinary importance comprise about more than forty species. Among these the most important tick species in Ethiopian cattle's are Amblyomma, Hyalomma, and Rhipicephalus (Boophilus) [8]. The country environmental condition and vegetation are highly conducive for ticks and tick-borne disease maintenance [5]. The life of ticks depends on the host animal which results in retardation of animal growth, loss of milk, and meat production, generally affecting the market and decreasing the annual income. Many people who live at rural area depend on the livestock production, which have faced to a considerable economic crisis due to tick infestation of cattle in the study area (source: district agricultural office). Tick infestation has been known to cause a great deal of loss or reduction of productivity by influencing the performance and qualities of the animal yield in the area which in turn leads to reduction of this sector contribution towards the country's development. Acaricide application is still the main method of tick control in Ethiopia [8]. Currently, organophosphates are the most widely used chemicals although evidence of resistance is emerging [9].

Although considerable amount of research has been done regarding ixodid ticks infestation in Ethiopia, it is still 


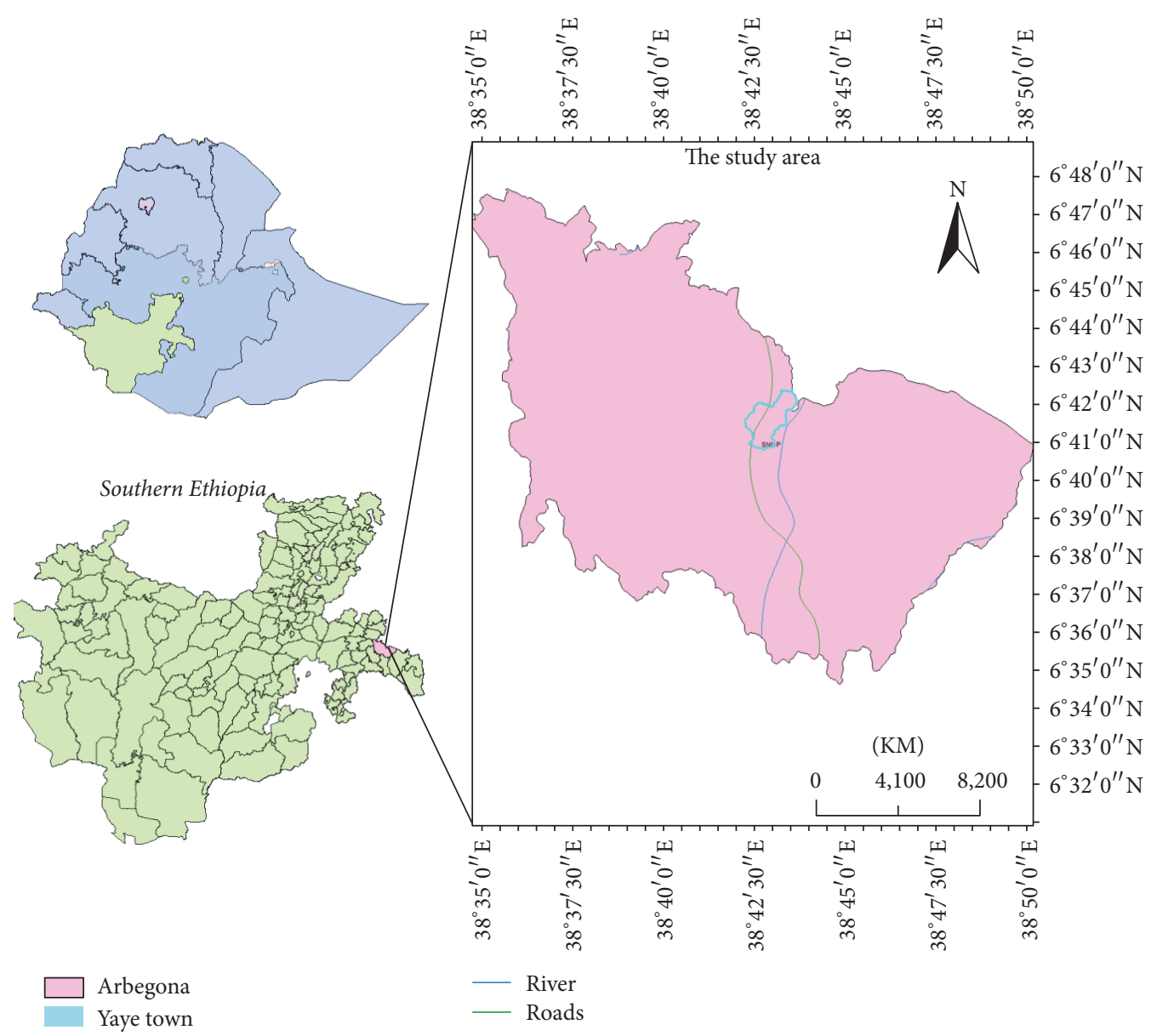

FIGURE 1: Map representing the study area (Arbegona district).

relevant to generate periodic and recent information about the prevalence of different species of ticks with the associated factors along different parts of the country. Consequently, minimizing the economic losses from tick infestation different studies in various parts of the country is needed so far. Furthermore, there was no known research conducted in the past and no any published information regarding tick infestation in cattle in the study area. The objectives of this study were aimed to identify and estimate the prevalence of various tick species and assess major factors that could contribute to tick infestation on cattle in the study area.

\section{Materials and Methods}

2.1. Description of the Study Area. The study was carried out in Arbegona district which is located at Sidama zone in southern Ethiopia about $344 \mathrm{~km}$ far from Addis Ababa, capital city of Ethiopia (Figure 1). Geographically, the district is located at $6^{\circ} 41^{\prime} \mathrm{N}$ latitude and $38^{\circ} 43^{\prime} \mathrm{E}$ longitude with an elevation of $8486 \mathrm{ft}$. Arbegona is bordered on the north by the Oromia Region, on the south by Bona Zuria, on the northwest by Gorche, on the southwest by Bursa, and on the east by Bensa. According to a 2004 central statistics agency report, Arbegona had 36 kilometers of all-weather roads and 25 kilometers of dry-weather roads. Climatically, Arbegona district belongs to the southern Ethiopia high land and it is mainly characterized by two agro ecological zones. These are mid-land (10\%) and arable high land (90\%). The district has 36 rural and 3 urban Kebeles (peasant associations (PA's) that usually consists of up to 200 households). Its altitude ranges from 2000 to 3300 meters above sea level (m.a.s.l.) with average rainfall ranging from 1600 to 1900 millimeter and the annual temperature varies between $7^{\circ} \mathrm{C}$ and $21^{\circ} \mathrm{C}$ [10]. Cattle are main assets and savings of the people and important source of protein and energy in their diet. Cattle by-products in rural areas mainly comes from indigenous zebu breed kept in traditional management system. Currently, cross breed cattle are increasing in number that primarily concentrate around urban and periurban areas where farmers supply their byproducts to urban consumers. In this district free grazing has been practiced over many generations predominantly located at valley bottoms or on wet lands favorable for tick infestation. The land of Arbegona is comparatively moist than the nearby districts of Sidama zone which has lowest cultivated crop coverage (100 hectare) since most of the land of this district is more than 2400 m.a.s.l. in the Moist-dega and in Moistwurch. It has also large open grazing land coverage. The land in the district is well covered with grass and vegetation and 
also reports show that some 4080 ha of the land is covered with bamboo forests.

2.2. Study Population. The study animals were cattle of all age, sex, breed, and body condition scores found in the three selected Kebeles of Arbegona district. The animals depend on grazing throughout the year for their feed sources with little supplementation of crop residues.

2.3. Study Design. A cross sectional study was conducted from October, 2014, to June, 2015, to estimate the prevalence of ticks, identification of the major ticks genera and species, their predilection sites, and burden along different age groups, breeds, season, body conditions, sex of animals, and different areas in the district. All the animals selected as sampling unit were checked for any tick infestation based on the number of ticks found on the animal and the study record period. Ticks were collected from ears, heads, dewlaps, belly/ flunk, udder/scrotum, fore/hind legs, perineum, and tails in the separated sample bottles with $70 \%$ ethyl alcohol (ethanol).

2.4. Method and Sources of Data Collection. The data for this study were collected from primary and secondary data sources. Questionnaires, interview, and observation as well as focus group discussion were used as primary sources to collect data from respondents concerning to tick infestation problems on the cattle's from their local area. All questionnaires were arranged in sequential manner that helps discussion and analysis. During the study period, 40 model farmers and animal health assistants from both sexes were considered for questionnaire survey to give their responses on major factors that lead cattle to tick infestation problem and 3 Kebeles were selected purposively according to accessibility and cattle population, proximity to livestock market, and other socioeconomic characteristics of the areas. Simple random sampling method was employed to select the study individual animals. The secondary data were collected by referring the district recorded document such as unpublished book and check list from the district agricultural office like case books concerning the general case on the tick infestation problem on the cattle. The age of the cattle was grouped into young (1 to 2 years), adult ( 3 to 7 years), and old ( $>8$ years) according to Gatenby [11] and Abera et al. [4], while body condition score was employed after categorizing the animals into poor, medium, and good according to Nicholson and Butterworth [12] after some modification. Extremely lean cattle, having prominent dorsal spines pointed to the touch and individual visible transverse processes into which a finger could be easily pushed, were considered as poor body condition score. A medium body condition score cattle was expressed as having usually visible ribs with little fat cover and barely visible dorsal spines. A good body condition score was given for the animals when fat cover easily seen in critical areas and felt and the transverse processes were not seen or felt. During the study, samples were collected in both dry and wet season of the year.
2.5. Sample Size Determination. The sample size was determined by using the formula given in Thrusfield [13]. Accordingly, with considering the expected prevalence of $50 \%$ and $5 \%$ absolute precision with $95 \%$ confidence interval, a total of 384 cattle were included in the study.

2.6. Tick Collection, Identification, and Count. The entire body surface of the animal was examined thoroughly for the presence of any tick and all visible adult ticks were collected from half-body on alternative sides. Ticks were removed carefully and gently in a horizontal pull to the body surface. The collected ticks were preserved in universal bottles containing $70 \%$ ethyl alcohol and labeled with the animal identification and predication site, age, sex, and data of collection. The specimens were transported to the parasitology laboratory of the school of veterinary medicine of Hawassa University for counting and identification. Ticks were counted and subsequently identified to genus and species level by using stereomicroscope, according to standard identification keys given by Walker et al. [7]. The half-body tick counts of cattle were doubled to obtain the whole body tick burdens. During examination of the selected animals for tick infestation, the age, sex, body condition score, breed, and Kebele of the sampled animals were recorded on a special format designed for this purpose. During the study, distribution of ticks and total count of each tick genera were done.

2.7. Data Analysis. The data were entered and managed in Microsoft-excel sheet. SPSS 20.0 version software program was employed for the data analysis. The overall prevalence of tick was determined by dividing the number of positive animals by total sample size and was expressed as percentage. Chi-square $\left(\chi^{2}\right)$ test was used to assess the association in tick infestation between different variables. Effects were reported as statistically significant in all cases if $p$ value is less than $5 \%$ $(p<0.05)$ and multivariable logistic regression was used to see the association of risk factors.

\section{Results}

3.1. Tick Infestation in Cattle and Different Risk Factors. Out of the total 384 cattle examined for the presence of ticks, 291 $(75.5 \%)$ were found to be infested with varying numbers of tick genera (Table 1). Higher tick prevalence was recorded in Charicho Kebele (85\%) and slightly lower prevalence in Gute $(68.7 \%)$ with no statistically significant difference $(p>$ $0.05)$. The occurrence of tick infestation in sex of animals was also not significantly different $(p>0.05)$. Tick infestation of animals with age and different body conditions showed that there were statistically significant variations $(p<0.05)$. During this study, there was higher prevalence of tick infestation in wet season (77.6\%) than dry season of the year (72.7\%) (Table 1).

3.2. Tick Burden and Species Identification. A total of 2024 adult ticks were collected from 291 cattle in the study sites (Table 2). Eight different tick species were registered from three genera including Boophilus subgenus of Rhipicephalus 
TABle 1: Potential risk factors for tick infestation status of cattle in Arbegona district.

\begin{tabular}{|c|c|c|c|c|c|}
\hline Risk factors & Number of animals examined & Number of positive animals & $95 \% \mathrm{CI}$ & $\chi^{2}$ & $p$ value \\
\hline \multicolumn{6}{|l|}{ Kebeles } \\
\hline Charicho & 128 & $109(85 \%)$ & - & \multirow{3}{*}{1.402} & \multirow{3}{*}{0.705} \\
\hline Gute & 128 & $88(68.7 \%)$ & $47.4-68.5$ & & \\
\hline Yaye 01 & 128 & $94(73.4 \%)$ & $53.8-73.4$ & & \\
\hline \multicolumn{6}{|l|}{$\operatorname{Sex}$} \\
\hline Female & 249 & $290(76.3 \%)$ & - & \multirow{2}{*}{0.559} & \multirow{2}{*}{0.454} \\
\hline Male & 135 & $101(74.8 \%)$ & $55.7-75.8$ & & \\
\hline \multicolumn{6}{|l|}{ Age } \\
\hline Old & 63 & $62(98.4 \%)$ & - & \multirow{3}{*}{6.154} & \multirow{3}{*}{0.046} \\
\hline Adult & 194 & $153(78.8 \%)$ & $56.7-68.0$ & & \\
\hline Young & 127 & $76(59.8 \%)$ & $42.4-68.8$ & & \\
\hline \multicolumn{6}{|c|}{ Body condition } \\
\hline Poor & 32 & $32(100 \%)$ & - & \multirow{3}{*}{6.812} & \multirow{3}{*}{0.000} \\
\hline Medium & 306 & $227(74 \%)$ & $46.6-58.9$ & & \\
\hline Good & 46 & $32(69.5 \%)$ & $69.1-100$ & & \\
\hline \multicolumn{6}{|l|}{ Season } \\
\hline Wet & 237 & $184(77.6 \%)$ & - & \multirow{2}{*}{1.023} & \multirow{2}{*}{0.312} \\
\hline Dry & 147 & $107(72.7 \%)$ & $51.1-67.9$ & & \\
\hline Total & 384 & $291(75.7 \%)$ & & & \\
\hline
\end{tabular}

Table 2: Proportion of ticks identified in Arbegona district.

\begin{tabular}{lc}
\hline Tick genera & Proportion \\
\hline Amblyomma & $708(34.9 \%)$ \\
Rhipicephalus (Boophilus) & $540(26.6 \%)$ \\
Hyalomma & $389(19.2 \%)$ \\
Rhipicephalus & $387(19 \%)$ \\
\hline Total & $2024(100 \%)$ \\
\hline
\end{tabular}

during the study period: two Amblyomma species (Figures 2(a) and 2(b)), two Rhipicephalus (Figures 2(c) and 2(d)), three Hyalomma (Figures 2(e), 2(f), and 2(g)), and Rhipicephalus (Boophilus) decloratus (Figure 2(h)).

3.3. Distribution of Tick Species on Body Parts of Study Animals. Amblyomma and Rhipicephalus (Boophilus) appeared to be dominant on the dewlap region of the animal while Hyalomma ticks prefer the sternum area next to dewlap of the animal. On the other hands, Rhipicephalus ticks tend to attach in the anal and tail section followed by ear area of the animal (Table 3).

3.4. Different Model Farmers and Animal Health Assistant Questionnaires. Over 87.5\% of the respondents acknowledged tick infestation of animal as problem while $35 \%$ of them know the presence of tick-borne diseases. Among the participant farmers, $65 \%$ of them recognize the effort of local veterinary workers toward minimizing and control of tick infestation while $55 \%$ of them believe that the beginning of rainy season favors tick infestation (Table 4).

\section{Discussion}

In the present study high overall prevalence of ticks $(75.7 \%)$ was registered (Table 1). Similarly, high prevalence of ixodid ticks was reported from different part of the country including $82 \%$ [14], 81.25\% [15], 74\% [16], and 65.5\% [17]. The high overall prevalence of tick infestation in cattle was also recorded by other authors such as Regassa [8] and Ayalew et al. [18] in the eastern and central part of Oromia, respectively. Similarly, higher finding was reported by de Castro [19] where it was stated that more than $80 \%$ of the cattle studied were ticks-infested. Abera et al. [4] reported around 95\% tick infestation prevalence in south western Ethiopia. Our study is not in line with the finding reported by Tiki and Addis [20] with a prevalence of $25.64 \%$. The inconsistency among these studies could be attributed to a wide range of factors including agroecological, animal health practice, or management difference with in their respective study areas. In this particular study, there is no significant difference $(p>$ 0.05) of tick infestation within three Kebeles of the district (Table 1). This is probably due to similarities in agroecological setting and animal health practice in these study sites.

In this study, Amblyomma was found to be the most abundant tick genera which accounts for $34.9 \%$ of the total finding. Likewise, Pawlos and Derese [21] indicated Amblyomma as the leading tick genera with $43.46 \%$ prevalence. This finding is also in agreement with that of previous reports on a high number of Amblyomma in three agroecological zones in central Oromia by Ayalew et al. [18] and at Haramaya University by Yehualashet et al. [22]. Amblyomma variegatum and Amblyomma cohearens were the two species of the genera identified during the study period in the area. Such finding echoes the alarming need of intervention since Amblyomma 

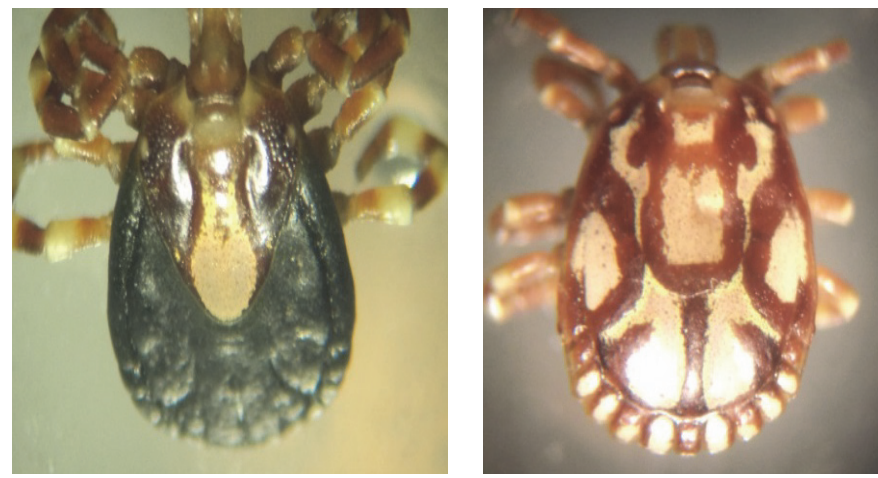

(a) Amblyomma variegatum (female at the left and male at right side)
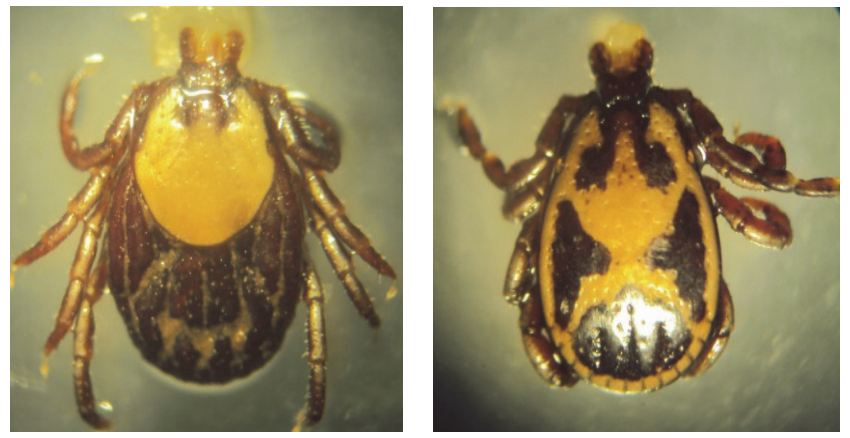

(c) Rhipicephalus pulchellus (female at the left and male at right side)
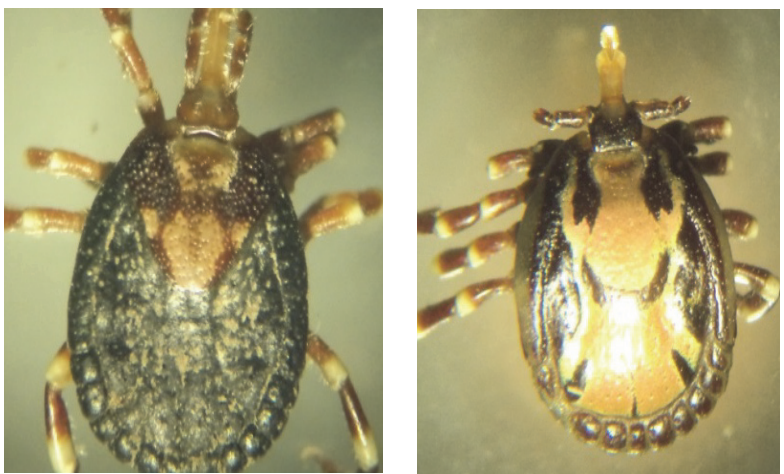

(b) Amblyomma cohaerens (female at the left and male at right side)
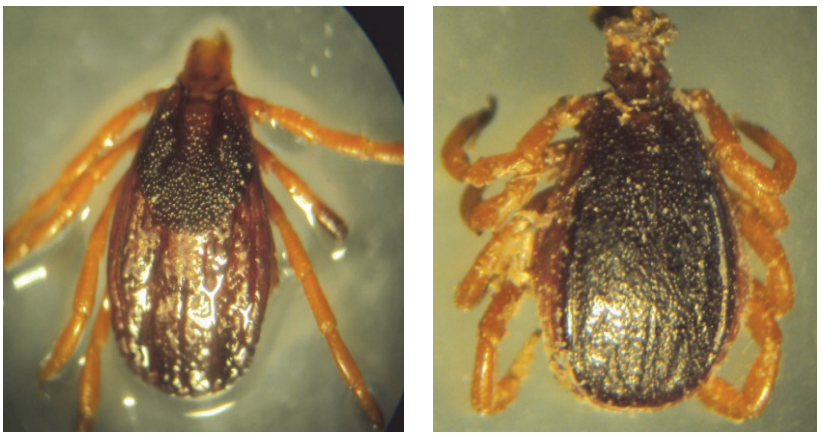

(d) Rhipicephalus evertsi evertsi (female at the left side and male at right side)

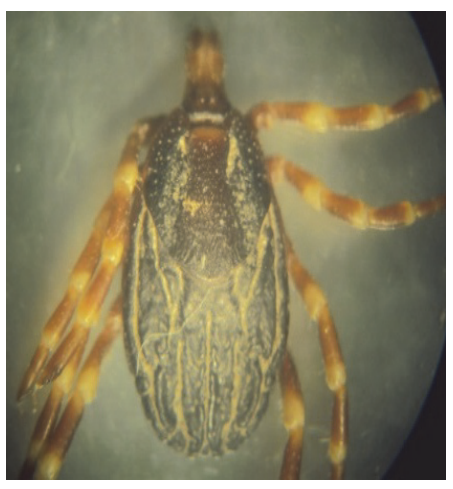

(e) Hyalomma impeltum (female dorsal)

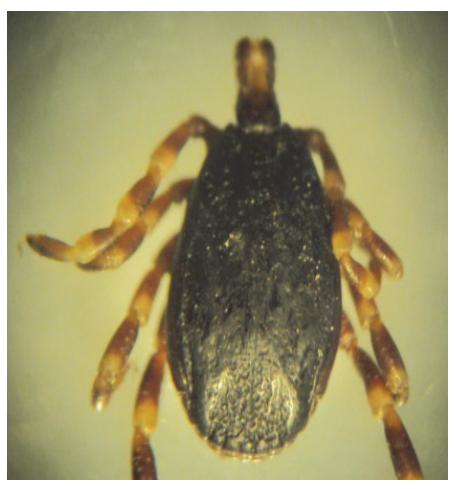

(f) Hyalomma truncatum (male dorsal)

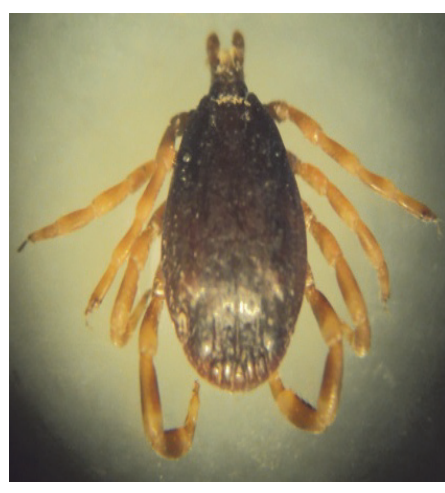

(g) Hyalomma anatolicum (male dorsal)

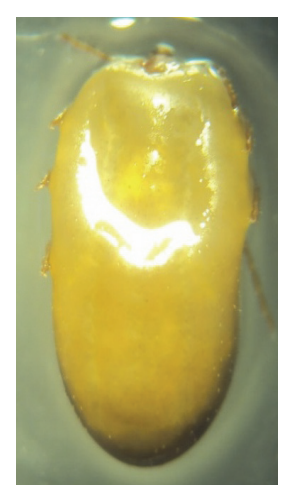

(h) Rhipicephalus (Boophilus) decloratus (female dorsal)

FIGURE 2: Representative pictures of identified different tick species of cattle during the study in the area.

TABLE 3: Genera of ticks and their distribution on body regions of cattle in Arbegona district.

\begin{tabular}{|c|c|c|c|c|c|}
\hline Body region & $\begin{array}{l}\text { Amblyomma } \\
\text { +ve (counted) }\end{array}$ & $\begin{array}{l}\text { Rhipicephalus (Boophilus) } \\
\text { +ve (counted) }\end{array}$ & $\begin{aligned} & \text { Hyalomma } \\
& \text { +ve (counted) }\end{aligned}$ & $\begin{array}{l}\text { Rhipicephalus } \\
\text { +ve (counted) }\end{array}$ & $\begin{array}{c}\text { Total } \\
\text { +ve (counted) }\end{array}$ \\
\hline Dewlap & $31(234)$ & $26(174)$ & $19(163)$ & $2(7)$ & $78(578)$ \\
\hline Udder & $28(226)$ & $17(114)$ & $9(35)$ & $3(9)$ & $57(384)$ \\
\hline Scrotum & $22(157)$ & - & $6(31)$ & $2(8)$ & $30(196)$ \\
\hline Anal region, under, tail & - & $20(131)$ & $7(14)$ & 25 (194) & $52(339)$ \\
\hline Sternum & $18(91)$ & $19(121)$ & 15 (146) & $3(11)$ & $55(369)$ \\
\hline Ear & - & - & - & $19(158)$ & $19(158)$ \\
\hline Total & $99(708)$ & $82(540)$ & $56(389)$ & $54(387)$ & $291(2024)$ \\
\hline
\end{tabular}


TABLE 4: Questionnaire data representing the question items and respondents response.

\begin{tabular}{|c|c|c|c|c|}
\hline \multirow{2}{*}{ Question contents } & \multirow{2}{*}{ Alternatives } & \multicolumn{3}{|c|}{ Respondents } \\
\hline & & Male & Female & Total (\%) \\
\hline \multirow{2}{*}{ Know the ticks } & Yes & 28 & 12 & $40(100)$ \\
\hline & No & - & - & $-(0)$ \\
\hline \multirow{2}{*}{ Tick problems in your locality } & Yes & 25 & 10 & $35(87.5)$ \\
\hline & No & 3 & 2 & $5(12.5)$ \\
\hline \multirow{3}{*}{ Type of ticks you know in your locality } & Hard tick & 16 & 8 & $24(60)$ \\
\hline & Soft tick & 4 & 2 & $6(15)$ \\
\hline & Both & 8 & 2 & $10(25)$ \\
\hline \multirow{3}{*}{$\begin{array}{l}\text { Type of ticks that seriously damage (affect) cattle in } \\
\text { your local area }\end{array}$} & Hard tick & 18 & 6 & $24(60)$ \\
\hline & Soft tick & 8 & 2 & $10(25)$ \\
\hline & Both & 4 & 2 & $6(15)$ \\
\hline \multirow{2}{*}{ Know the season of tick infestation outbreak } & Yes & 28 & 12 & $40(100)$ \\
\hline & No & - & - & $-(0)$ \\
\hline \multirow{4}{*}{ Season of tick infestation outbreak } & $\begin{array}{l}\text { At the end of } \\
\text { rain season }\end{array}$ & 2 & 1 & $3(7.5)$ \\
\hline & $\begin{array}{l}\text { At the } \\
\text { beginning of } \\
\text { rainy season }\end{array}$ & 16 & 6 & $22(55)$ \\
\hline & $\begin{array}{l}\text { In mid of rainy } \\
\text { season }\end{array}$ & 4 & 3 & $7(17.5)$ \\
\hline & $\begin{array}{l}\text { At the dry } \\
\text { season }\end{array}$ & 6 & 2 & $8(20)$ \\
\hline \multirow{2}{*}{ Know any tick-borne disease } & Yes & 10 & 4 & $14(35)$ \\
\hline & No & 18 & 8 & $26(65)$ \\
\hline \multirow[b]{2}{*}{ Species of livestock mostly infected by ticks } & Bovine & 28 & 12 & $40(100)$ \\
\hline & $\begin{array}{l}\text { Ovine, caprine, } \\
\text { others }\end{array}$ & - & - & - \\
\hline \multirow{2}{*}{$\begin{array}{l}\text { Breed of cattle comparatively more susceptible to the } \\
\text { tick infestation in your locality }\end{array}$} & $\begin{array}{l}\text { Indigenous } \\
\text { breed }\end{array}$ & 9 & 8 & $17(42.5)$ \\
\hline & Cross breed & 19 & 4 & $23(57.5)$ \\
\hline \multirow{2}{*}{$\begin{array}{l}\text { District veterinary workers contribute to minimize and } \\
\text { control the prevalence of tick and tick infestation }\end{array}$} & Yes & 18 & 8 & $26(65)$ \\
\hline & No & 10 & 4 & $14(35)$ \\
\hline
\end{tabular}

ticks are a potential vector for a disease caused by Cowdria rumintium [23] which is common where this tick is prevalent in the country. Rhipicephalus (Boophilus) was the second most abundant tick subgenus (26.6\%) in this study. Mekonnen et al. [23] described Rhipicephalus (Boophilus) as the commonest and most wide spread tick in Ethiopia. Similar to the current finding, Abebe et al. [24] reported Rhipicephalus (Boophilus) decoloratus (24.83\%) as the second abundant tick species while Tessema and Gashaw [25] indicated 15.4\% prevalence. On the contrary, the findings of Alekaw [26] at Metekel Ranch, Ethiopia, showed a lower prevalence $(5.7 \%)$ of Rhipicephalus (Boophilus) tick species. This may be due to the geographical location and altitude factors which belongs to lower area of the country with 1500 to 1600 m.a.s.l. of Metekel ranch. The identified tick species of this genera were Rhipicephalus pulchellus, Rhipicephalus evertsi evertsi, and Rhipicephalus (Boophilus) decloratus. Female Rhipicephalus (Boophilus) were abundant from September to April and could transmit Babesia bigemina, in addition to anorexia and anemia in case of severe infestation [27]. Season-wise, Shiferaw [28] indicated that Rhipicephalus (Boophilus) had highest frequency during dry seasons (January, February, and early March) in the observed area of Wolaita Zone.

Hyalomma is the third most abundant tick genera (19.2\%) in this study which is in compatible with Tessema and Gashaw [25]. Likewise, Getachew et al. [15] reported Hyalomma as the second most abundant tick with $20.34 \%$ prevalence. Lower prevalence $(5 \%)$ of $H$. m. rufipes was reported by Ayalew et al. [18] from Sebeta Waso District. In the current study, the fourth and least abundant tick genera were Rhipicephalus having a prevalence of $19 \%$. This finding was close to the works conducted by Mekonnen et al. [23] at Ghibe Tullary in central Ethiopia who reported $21.2 \%$ prevalence. The finding is also consistent with Ayalew et al. [18] who found $19.5 \%$. This tick genus shows no apparent preference for any particular altitude, rainfall, or season [5]. Hyalomma impeltum, 
Hyalomma truncatum, and Hyalomma anatolicum were tick species of this genera identified in the study area which are possible and potential vectors for Babesia, Rickettsia, and Theleria diseases [29].

With regard to distribution pattern and predilection site of ticks, Amblyomma and Boophilus had relatively fair distribution to almost all the examined body regions of animals. Rhipicephalus ticks were restricted to the anal region and under tail and ear areas, with very few of them observed on the scrotal, udder, dewlap, and sternum area which is also true in the work of Nateneal et al. [14] who reported finding of Rhipicephalus evertsi evertsi exclusively in perineum and anal area.

In the present study, among the considered variables as a factor for tick prevalence, only age and body condition groups had significant association $(p<0.05)$ with prevalence of tick. Tick burden was significantly $(p<0.05)$ higher in older animals than the other age groups. This is probably associated with low immunity and resistance of old animals. Regarding body condition, animals with poor body condition showed significantly $(p<0.05)$ higher tick infestation than the other groups. This may be due to the fact that poorly conditioned animals had low resistant to tick infestation and lack enough body capacity to build resistance whereas animals with good body condition showed reasonable combat to the infestation according to [30]. On the way, tick infestation might be a cause for poor body condition instead of vice versa.

In the current study, there was no considerable difference $(p>0.05)$ in the prevalence of ticks within the wet and dry season. However, Mohamed et al. [31] report indicates significantly $(p<0.05)$ increased prevalence of tick in wet season than dry season. Mekonnen et al. [23] reported that ticks were found on cattle throughout the study period, although higher tick counts were observed during the rainy than dry season. The most important environmental factors that influence the occurrence of ticks in a biotope include climate such as temperature and relative humidity [32]. Even if the same factor affects the survival of all tick species to varying degrees, each tick species has its particular threshold temperature and moisture during their life time. The survival of ticks also depends on the presence of hosts suitable for reproduction by the adults [7]. For instance, Rhipicephalus (Boophilus) species are adapted to feed on cattle, but some may survive by feeding on sheep or antelope.

The present questionnaire survey result revealed that the entire respondents' know or had information about the ticks. $87.5 \%$ of the interviewed participant believe that there was tick infestation problems in livestock in their locality. Ticks affect livestock in general and cattle in particular by reducing milk production, growth, hide and skin, and birth rate. From the total respondents interviewed, $60 \%$ confirmed that hard ticks are more common and affect livestock productivity in their locality. The same percentage of respondents also identified hard tick with that of soft ticks infesting cattle. According to the respondents analysis, tick infestation occurs throughout the year, but majority of them described tick infestation was most favored at the beginning of rain season followed by the dry and mid rain season with lower prevalence at the end of rain season. Comparable findings were recorded in Jimma Zone [33] and in Borena pastoral area $[34,35]$. The questionnaire survey finding indicated only $35 \%$ of the participants know tick-borne diseases transmitted by ticks while the remaining $65 \%$ revealed that they do not know tick-borne diseases. This showed effects and constraints of ticks in livestock productivity and health impact has been not well understood by the community in the area. The present study also revealed that cross breed cattle are comparatively more susceptible to tick infestation compared to indigenous breed cattle in their locality. Similar result was also reported by other researchers in the country with high susceptibility of cross or exotic breed cattle than local breeds to tick infestation [36]. In the present questionnaire survey, $65 \%$ of the respondents acknowledge the involvement of district veterinary workers in the control of tick infestation even though there is no well-planned program of tick control strategy in the study site in particular and the country in general except on dairy farms [37].

\section{Conclusion}

The study demonstrated that there was high burden of ticks in the area with overall prevalence of $75.5 \%$ which indicates ticks are common and important ectoparasite of cattle in Arbegona district. This study showed that there was high burden and prevalence of ticks that still play major roles in reducing productivity and cause health problems of cattle in the area. Further detailed studies on the role of different ticks species in causing disease in cattle and their economic consequence to the livelihoods call for urgent attention.

\section{Competing Interests}

The authors declare that there is no conflict of interests regarding the publication of this paper.

\section{Acknowledgments}

The authors would like to thank Hawassa University School of Veterinary Medicine Laboratory workers and Arbegona district veterinary clinic members for their all-round helps during the work.

\section{References}

[1] Central Statistical Agency (CSA), Agricultural Sample Survey. Report on Livestock and Livestock Characteristics (Private Peasant Holdings), vol. 1, Central Statistical Agency (CSA), Addis Ababa, Ethiopia, 2015.

[2] H. T. Yacob, H. Ataklty, and B. Kumsa, "Major ectoparasites of cattle in and around Mekelle, northern Ethiopia," Entomological Research, vol. 38, no. 2, pp. 126-130, 2008.

[3] W. Asfaw, "Country report: Ethiopia," in Proceedings of the Seminar on Livestock Development Policies in Eastern and South Africa, Organization by CTA, OAU, the Ministry of Agriculture and Cooperative, Mbabane, Swaziland, 1997.

[4] M. Abera, T. Mohammed, R. Abebe, K. Aragaw, and J. Bekele, "Survey of ixodid ticks in domestic ruminants in Bedelle 
district, Southwestern Ethiopia," Tropical Animal Health and Production, vol. 42, no. 8, pp. 1677-1683, 2010.

[5] R. G. Pegram, Hoogstraal, and H. Y. Wassef, “Ticks (Acari: Ixodidae) of Ethiopia. Distribution, Ecology and relation of species infesting livestock," Bulletin of Entomology Research, vol. 71, pp. 335-359, 1981.

[6] S. Rahbori, P. Shvan, and R. Haddodzadeh, "Domestic ruminants in Iran, Korean, Journal of Parasitology," 2009, https://www .ncbi.nlm.nih.gov/pmc/articles.

[7] A. A. Walker, A. Bouatour, J. L. Camicas et al., Ticks of Domestic Animals in Africa: A Guide to Identification of Species, The University of Edinburgh, Edinburgh, UK, 2003.

[8] A. Regassa, "The use of herbal preparations for tick control in western Ethiopia," Journal of the South African Veterinary Association, vol. 71, no. 4, pp. 240-243, 2000.

[9] J. Yilma, G. Adamu, and E. Zerbini, "Biossay of acaricide resistance on three common cattle tick species at Holotta, Central Ethiopia," Revue de Médecine Vétérinaire, vol. 152, no. 5, pp. 385-390, 2001.

[10] Central Statistical Authority (CSA), Agriculture Sample Survey. Report on Livestock Population, Private and Peasant Holding, vol. 1, Central Statistical Authority (CSA), Addis Abeba, Ethiopia, 2004.

[11] R. Gatenby, The Tropical Agriculture, McMillan Education, London, UK, 1991.

[12] M. Nicholson and T. Butterworth, A Guide to Body Condition Score in Zebu Cattle International Livestock Center for Africa, 1986.

[13] M. V. Thrusfield, Veterinary Epidemiology, Blackwell Science, London, UK, 3rd edition, 2005.

[14] T. Nateneal, E. Fikadu, M. Yimer, and K. Jelalu, "Identification and prevalence of ixodid tick in bovine at Bedele district, Oromiyia Regional State, Western Ethiopia," Journal of Parasitology and Vector Biology, vol. 7, no. 8, pp. 156-162, 2015.

[15] A. Getachew, C. Mersha, M. Dessalegne, and B. Basaznew, "Prevalence of ixodid ticks on cattle in Northwest Ethiopia," Acta Parasitologica Globalis, vol. 5, no. 2, pp. 139-145, 2014.

[16] G. Meaza, M. Abdu, and K. Yisehak, "Determination of the prevalence of ixodid ticks of cattle breeds, their predilection sites of variation and tick burden between different risk factors in Bahir Dar, Ethiopia," Global Veterinaria, vol. 13, no. 4, pp. 520-529, 2014.

[17] A. Wolde and A. Mohamed, "Prevalence of ixodid ticks on Bovine in Soddozuria districts, Wolaita Zone, Ethiopia," Acta Parasitologica Globalis, vol. 5, no. 3, pp. 188-197, 2014.

[18] T. Ayalew, Y. Hailu, and B. Kumsa, "Ixodid ticks infesting cattle in three agroecological zones in central Oromia: species composition, seasonal variation, and control practices," Comparative Clinical Pathology, vol. 23, no. 4, pp. 1103-1110, 2014.

[19] J. J. de Castro, "Tick survey. A survey of the tick species in Western Ethiopia," Tech. Rep. AG:DP/ ETH/83/023, Food and Agricultural Organization of the United Nations (FAO), Rome, Italy, 1994.

[20] B. Tiki and M. Addis, "Distribution of ixodid ticks on cattle in and around holeta town, Ethiopia," Global Veterinaria, vol. 7, no. 6, pp. 527-531, 2011.

[21] W. Pawlos and D. Derese, "Study on prevalence and identification of ticks in Humbo district, Southern Nations, Nationalities, and People's Region (SNNPR) Ethiopia," Journal of Veterinary Medicine and Animal Health, vol. 5, no. 3, pp. 73-80, 2013.
[22] T. Yehualashet, F. Gebreab, A. Wakjira, and T. Tsega, "Preliminary observation on ticks, Seasonal dynamics and resistance of three indigenous and three crossbred cattle in Ethiopia," Bulletin of Animal Health and Production in Africa, vol. 43, p. 114, 1995.

[23] S. Mekonnen, R. G. Pegram, S. Gebre, A. Mekonnen, M. Jobre, and S. Zewde, "A synthesis review of ixodid (Acari: Ixodidae) and Argasid (Acari: Argasidae) ticks in Ethiopia and their possible roles in disease transmission," Ethiopian Veterinary Journal, vol. 11, no. 2, pp. 1-25, 2007.

[24] R. Abebe, T. Fantahun, M. Abera, and J. Bekele, "Survey of ticks (Acari: Ixodidae) infesting cattle in two districts of somali regional state, Ethiopia," Veterinary World, vol. 3, no. 12, pp. 539$543,2010$.

[25] T. Tessema and A. Gashaw, "Prevalence of ticks on local and crossbred cattle in and around Asella town, southeast Ethiopia," Ethiopian Veterinary Journal, vol. 14, no. 2, pp. 79-89, 2011.

[26] S. Alekaw, "Distribution of ticks and tick-borne diseases at Metekel Ranch," Ethiopian Veterinary Journal, vol. 4, no. 1, p. 30, 1998.

[27] Z. Seyoum, Distribution and Host Parasite Relationship of Ixodids Ticks in Eastern Amhara, Kombolcha Regional Veterinary Laboratory, Kombolcha, Ethiopia, 2005.

[28] D. Shiferaw, Cattle tick dynamics in different agro-ecological zones of Wolaita, Southern Ethiopia [M.S. thesis], Faculty of Veterinary Medicine, Addis Ababa University, Debre Zeit, Ethiopia, 2005.

[29] D. S. Kettle, Medical and Veterinary Entomology, CAB International, London, UK, 2nd edition, 1995.

[30] A. Manan, Z. Khan, B. Ahmad, and Abdullah, Prevalence and Identification of Ixodid. Ministry of Economic Development and Cooperation Survey of Livestock and Fisheries Development, Agricultural Development Department, Livestock Team, Addis Ababa, Ethiopia, 2007.

[31] B. Mohamed, A. Belay, and D. Hailu, "Species composition, prevalence and seasonal variations of ixodid cattle ticks in and around Haramaya town, Ethiopia," Journal of Veterinary Medicine and Animal Health, vol. 6, no. 5, pp. 131-137, 2014.

[32] A. A. Latif and A. R. Walker, "An introduction to the biology and control of ticks in Africa," ICTTD-2 Project, 2004.

[33] G. Abebaw, "Seasonal dynamics and host preference of Boophilus decoloratus on naturally infested cattle in Jimma zone, south western Ethiopia," Ethiopian Veterinary Journal, vol. 18, no. 1, pp. 19-28, 2004.

[34] T. Sori, Ethno Veterinary Practice of Borana Pastoralist and Their Possible Contribution to Livestock Production and Management, National Veterinary Institute (NVI), Debre Ziet, Ethiopia, 2004.

[35] CARE-Ethiopia, Value Chain Analysis of Milk and Milk Products in Borana Pastoralist Area, Regional Resilience Enhancement against Drought Project, Yonas Business Promotion and Consultancy P LC, Addis Ababa, Ethiopia, 2009.

[36] D. Ayana, E. Eshetu, H. Waketole, and F. Abunna, "In-vitro acaricidal efficacy evaluation trial of Ixodid ticks at Borana, Ethiopia," Ethiopian Veterinary Journal, vol. 17, no. 2, pp. 85-99, 2013.

[37] S. Mekonnen, "In vivo evaluation of amitraz against ticks under field conditions in Ethiopia," Journal of the South African Veterinary Association, vol. 72, no. 1, pp. 44-45, 2001. 

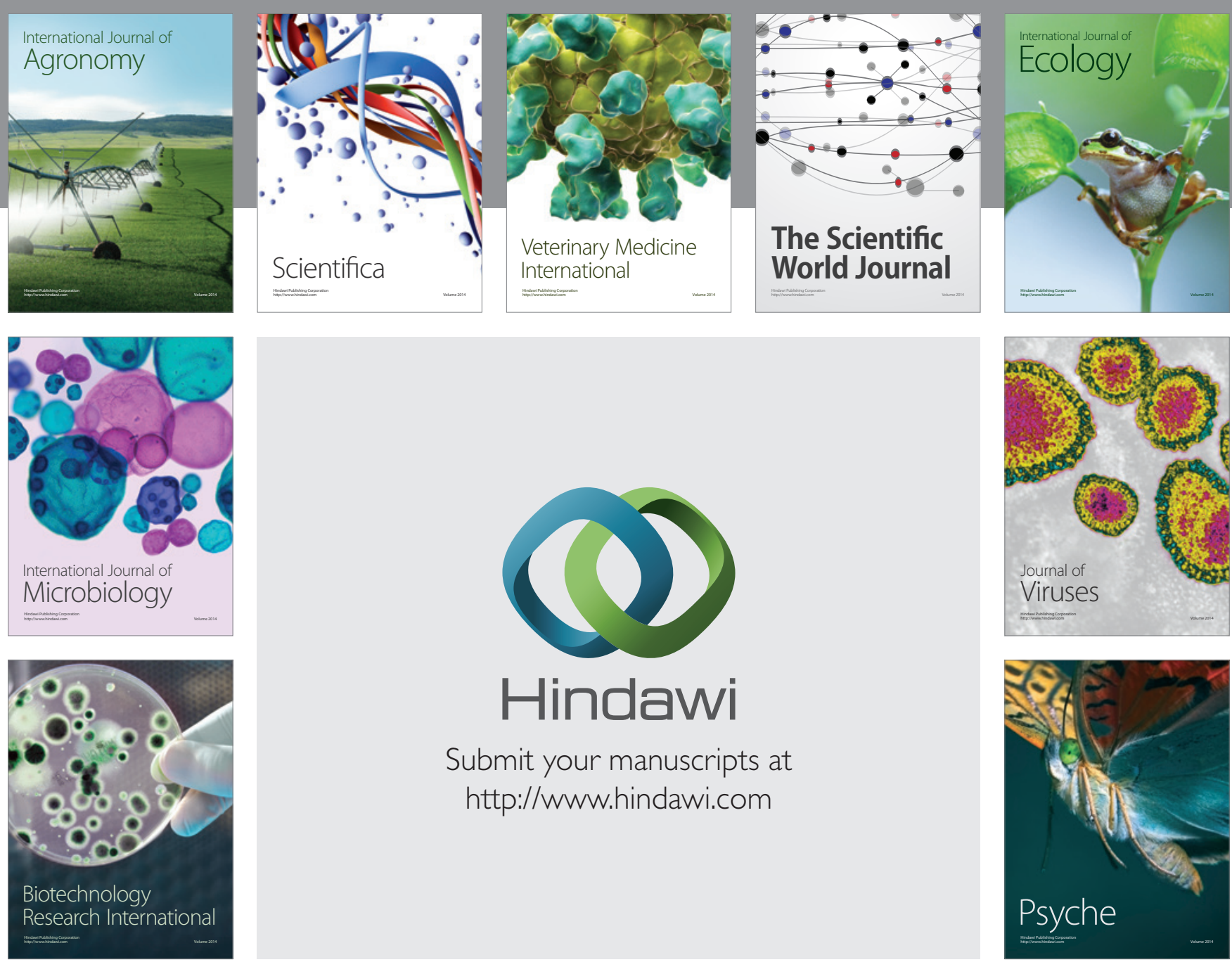

Submit your manuscripts at

http://www.hindawi.com
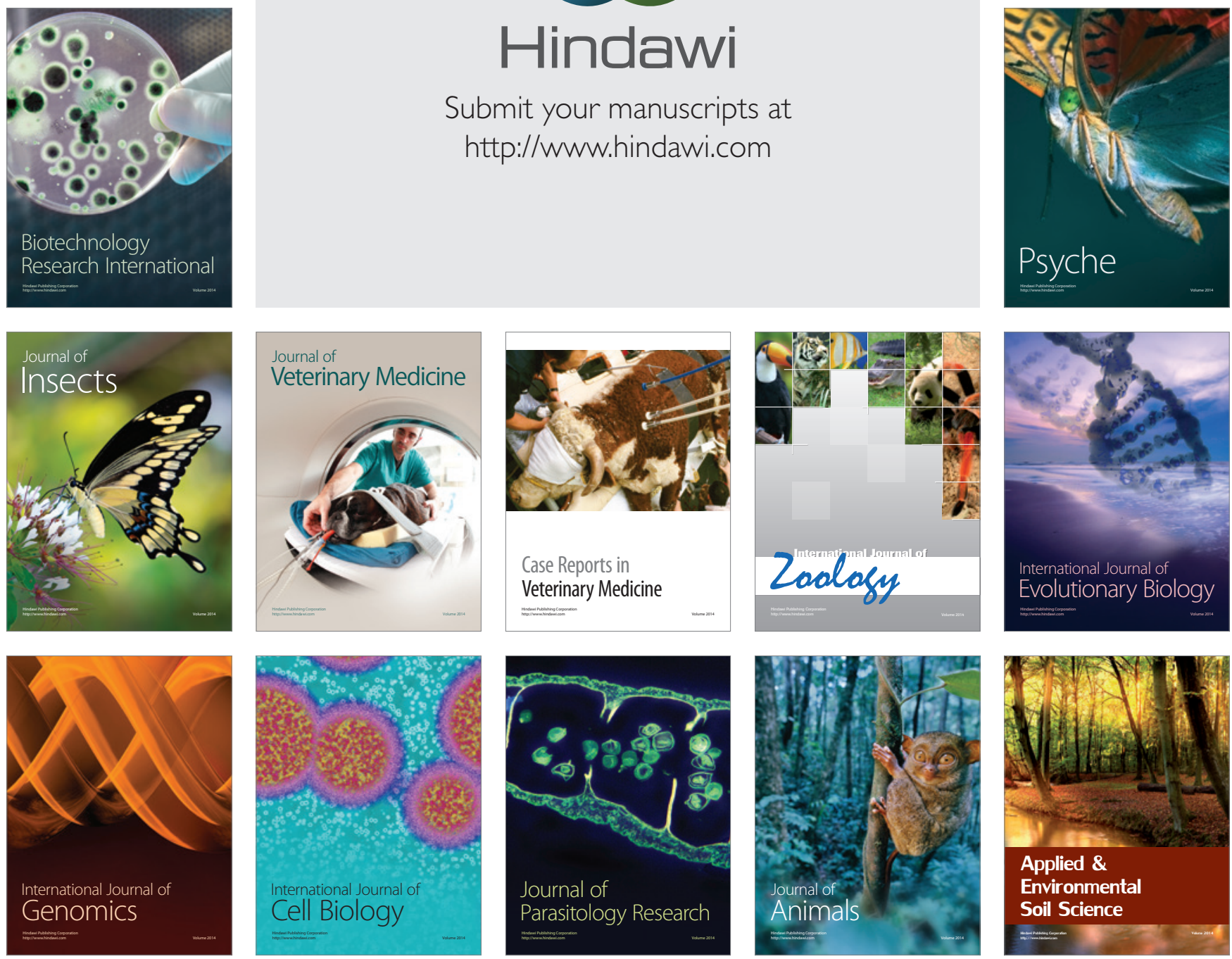